\title{
Electronic and magnetic properties of the interface between metal-quinoline molecules and cobalt
}

\author{
Andrea Droghetti, ${ }^{1}$ Sabine Steil, ${ }^{2}$ Nicolas Großmann, ${ }^{2}$ Norman Haag, ${ }^{2}$ Hongtao Zhang, ${ }^{3}$ Maureen Willis, ${ }^{4}$ William P. Gillin, ${ }^{3,4}$ \\ Alan J. Drew, ${ }^{3}$ Martin Aeschlimann, ${ }^{2}$ Stefano Sanvito, ${ }^{1}$ and Mirko Cinchetti ${ }^{2}$ \\ ${ }^{1}$ School of Physics, AMBER and CRANN Institute, Trinity College, Dublin 2, Ireland \\ ${ }^{2}$ Department of Physics and Research Center OPTIMAS, University of Kaiserslautern, Erwin-Schroedinger Strasse 46, \\ 67663 Kaiserslautern, Germany \\ ${ }^{3}$ Materials Research Institute and School of Physics and Astronomy, Queen Mary University of London, Mile End Road, \\ E1 4NS London, United Kingdom \\ ${ }^{4}$ College of Physical Science and Technology, Sichuan University, Chengdu 610064, People's Republic of China \\ (Received 9 December 2013; revised manuscript received 18 February 2014; published 12 March 2014)
}

\begin{abstract}
It was recently established that spin injection from a ferromagnetic metal into an organic semiconductor depends largely on the formation of hybrid interface states. Here we investigate whether the magnetic properties of the interface between cobalt and tris(8-hydroxyquinolinato)- $\mathrm{Al}(\mathrm{III})\left(\mathrm{Alq}_{3}\right)$, the most prominent molecular candidate for organic spin-valve devices, can be modified by substituting the aluminum atom with either gallium or indium. The electronic structure of $\mathrm{Alq}_{3}, \mathrm{Gaq}_{3}$, and $\mathrm{Inq}_{3}$ and the properties of their interfaces with ferromagnetic cobalt are probed experimentally, by using different photoemission spectroscopy methods, and theoretically, through density functional theory calculations. For all cases, the results highlight the presence of spin-polarized interface states. However no striking difference between the properties of the various molecules and interfaces is observed. This is a consequence of the fact that the molecules frontier orbitals are mainly localized on the ligands and they show only a negligible contribution coming from the metal ion.
\end{abstract}

DOI: 10.1103/PhysRevB.89.094412

PACS number(s): 72.25.Mk, 71.20.Rv, 79.60.Fr, 79.60.Dp

\section{INTRODUCTION}

Since the first report of magnetoresistence in organic semiconductors (OSCs) [1], the field of organic spintronics [2,3] has grown quickly and has witnessed a rush towards the fabrication of novel hybrid organic-inorganic (HOI) spin-valve devices [4]. However, unfortunately, the interpretation of the experimental results is often puzzling and the performances of such devices are hardly reproducible. This can be partially ascribed to the incomplete understanding of the main mechanism, which governs spin and charge injection from a ferromagnetic metal into an OSC and across the HOI interfaces.

Charge transport through HOI interfaces has been widely investigated due to its relevance for organic electronics and photovoltaic technologies. It is now established that the electron/hole transport across a HOI interface sets in whenever an applied bias shifts the lowest unoccupied/highest occupied molecular orbital (LUMO/HOMO) to the metal Fermi energy, thus overcoming a potential barrier [5-7], which is typically a few hundreds meV high $[8,9]$. Unfortunately this picture, which has proven quite successful in elucidating charge transport processes, does not describe spin-polarized injection from a ferromagnetic electrode into an OSC. In fact, because of the potential barrier, spin injection would not be possible at very low bias voltages and low temperature, which, instead, are the typical working conditions for large magnetoresistance in HOI spin valves [4]. More drastically, although spin injection was unequivocally demonstrated in several experiments $[10,11]$, neither theoretical models nor phenomenological arguments are currently able to explain what are the main parameters affecting its efficiency.

In this context, it was recently suggested that spin injection is a process deeply related to the formation of hybrid interface states [12,13], which can be spin polarized with the sign and the absolute value of the spin polarization determined by the strength and by the character of hybridization between the OSCs molecular orbitals and the spin-polarized bands of the ferromagnetic surface. Then, such hybrid states, which have been clearly visualized through photoemission [14-16] and scanning tunneling microscopy experiments [17-19], act as a further spin filter [14] so that the spin polarization of the current injected into an OSC can be very different from that of the ferromagnetic electrodes and it may sometimes have even opposite sign. On the one hand, the large dependence of the magnetic and transport properties of the HOI interfaces on both the strength and nature of the molecule-metal hybridization may pose new challenges for a deep understanding of the experimental results. On the other hand, the possibility to modify OSCs through chemical functionalization [20] may offer new unexpected routes for engineering the spin-transport properties of HOI interfaces $[21,22]$. This intriguing idea was investigated, for example, by Lach et al. [15], who described how the spin selectivity of the cobalt surface can be drastically tuned through the chemisorption of various metal phthalocyanines.

We here investigate, both theoretically and experimentally, the electronic structure of the interface between epitaxial Co thin films grown on $\mathrm{Cu}(001)$ and three tris(8hydroxyquinolinato)-metal molecules $\mathrm{Mq}_{3}$, which differ only by the metal center $M=\mathrm{Al}$, In, and Ga (Fig. 1). We then aim at understanding whether and how the various metal ions affect the magnetic properties of the interface.

$\mathrm{Alq}_{3}$ is one of the most popular OSCs used as an active layer in organic light-emitting diodes and, moreover, it represents the prototypical OSC employed in organic spin valves. $\mathrm{Gaq}_{3}$ and $\mathrm{Inq}_{3}$ have recently been synthesized [23]. Although they have been previously studied and implemented in light-emitting devices [24-26], they may also represent interesting novel OSCs for spintronics. In fact, on the one 


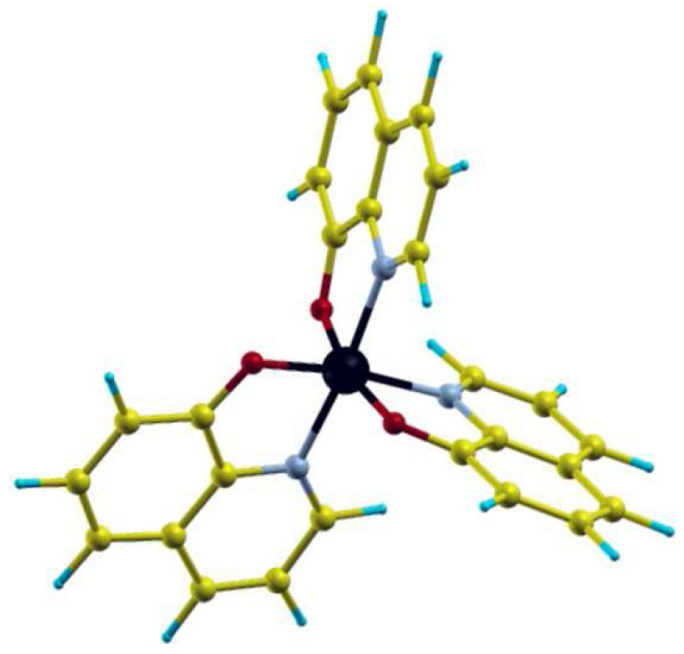

FIG. 1. (Color online) Structure of the $\mathrm{Mq}_{3}$ molecule. Color code: C (yellow), O (red), N (gray), H (blue), and $M$ (Al, Ga, and In) (black).

hand, the different relative atomic radius of the three metal ions may affect their phononic properties, which, ultimately, would reflect in the polaron transport mechanism and thus in typical devices characteristic behavior. On the other hand, the different atomic number of $\mathrm{Al}, \mathrm{Ga}$, and In modifies the spin-orbit interaction strength, affecting the spin-relaxation time [23]. Our present study extends a previous work [20], in which the properties of $\mathrm{Alq}_{3}$ molecules were compared to those of the related compound tris-(9-hydroxyphenalen1oate)-aluminum(III) $\left[\mathrm{Al}(\mathrm{OP})_{3}\right] . \mathrm{Al}(\mathrm{OP})_{3}$, while having the same metal center as $\mathrm{Alq}_{3}$, is characterized by much bigger ligands bounded to the $\mathrm{Al}^{3+}$ ion only via oxygen donor atoms.

By combining ultraviolet-photoemission spectroscopy (UPS), spin-resolved near-threshold photoemission spectroscopy (NT-PS), spin-resolved two-photon photoemission (2PPE) spectroscopy, and state-of-the-art density functional theory (DFT) calculations, our study unequivocally shows that all three $\mathrm{Mq}_{3}$ molecules bind so strongly to the surface that spin-polarized interface states are formed. However, the interface electronic structure is very similar in all cases, which means that the spin-filtering properties of the interfaces cannot be engineered by simply substituting $\mathrm{Al}$ with Ga or In.

The paper is organized as follows: First we describe both the experimental and theoretical methods employed (Secs. II and III). Then, after introducing the electronic structure of $\mathrm{Alq}_{3}, \mathrm{Gaq}_{3}$, and $\mathrm{Inq}_{3}$ (Sec. IV), we discuss the properties of their interfaces with Co providing a detailed analysis of the magnetic properties (Secs. V and VI). Finally we summarize the main results and conclude (Sec. VII).

\section{EXPERIMENTAL METHODS}

Spectroscopic measurements were performed in a UHV system consisting of one spectrometer chamber and two evaporator chambers. The base pressure in the spectrometer chamber is $4 \times 10^{-11}$ mbar. The evaporator chambers enable one to produce the $\mathrm{Co} / \mathrm{Mq}_{3}$ systems in situ, which is crucial to obtain a clean surface and a high-quality interface. The $3.5 \mathrm{~nm}$ thin Co films were deposited by electron beam epitaxy with an Omicron EFM-3 evaporator on a $\mathrm{Cu}(001)$ single crystal at a pressure of $10^{-10}$ mbar. Afterwards the sample was annealed at $370 \mathrm{~K}$. This results in a metastable tetragonally distorted Co fcc structure with an in-plane magnetic uniaxial anisotropy along the (110) direction of copper [27]. $\mathrm{Alq}_{3}$ (99.995\% pure) was purchased from Sigma Aldrich. $\mathrm{Gaq}_{3}$ and $\mathrm{Inq}_{3}$ were synthesized using a published method [28,29]. All three $\mathrm{Mq}_{3}$ were purified using train sublimation, resulting in a polycrystalline powder. After purification, the $\mathrm{Mq}_{3}$ are deposited on the freshly prepared cobalt surface with a Knudsen cell from Kentax $\mathrm{GmbH}$ at a pressure of $9 \times 10^{-10}$ mbar. The deposition rates are monitored by a quartz crystal balance calibrated with ellipsometry.

To detect the occupied manifold of the $\mathrm{Co} / \mathrm{Mq}_{3}$ system we performed UPS and spin-resolved NT-PS [30]. UPS was performed by using an Omicron HIS 13 vacuum ultraviolet lamp, which operates at the He Iline $(h v=21.2 \mathrm{eV})$. The excitation source for NT-PS is a Ti:sapphire laser system with a central wavelength of $800 \mathrm{~nm}, 82 \mathrm{MHz}$ repetition rate, a pulse power of $1.5 \mathrm{~W}$, and a pulse duration of $100 \mathrm{fs}$. The output is frequency quadrupled by using two $\beta$-barium borate crystals leading to the fourth harmonic of the fundamental with a photon energy of $h v=5.95 \mathrm{eV}$. For both UPS and NT-PS the light incident angle was $45^{\circ}$. In order to detect the unoccupied manifold of the $\mathrm{Co} / \mathrm{Mq}_{3}$ system we performed spin-resolved 2PPE. The setup for the 2PPE experiments is reported in Ref. [14]; the photon energy used was $3.26 \mathrm{eV}$ and the pulse length was 63 fs. In the UPS, NT-PS, and 2PPE experiments the emitted photoelectrons are analyzed in energy and spin by a Focus SPLEED detector mounted on a cylindrical sector analyzer (Focus CSA). The acceptance angle of the detector system is $\pm 13^{\circ}$ and the energy resolution is $0.22 \mathrm{eV}$. All presented measurements were performed at room temperature.

\section{COMPUTATIONAL DETAILS}

DFT calculations were performed by using a development version of the SIESTA code [31]. Norm-conserving Troullier-Martin pseudopotentials were employed together with a basis set of double- $\zeta$ plus polarization quality. In the case of $\mathrm{Gaq}_{3}$ and $\mathrm{Inq}_{3}$, the $3 d$-Ga and $4 d$-In states were included as valence orbitals. The Perdew-Burke-Ernzerhof (PBE) generalized gradient approximation (GGA) [32,33] for the exchange-correlation functional was used. However, the density of states (DOS) of the molecules was also computed with the atomic self-interaction correction (ASIC) scheme [34,35]. In fact, for the free $\mathrm{Alq}_{3}$ molecule, ASIC is able to return a density of (occupied) states, which closely matches both the UPS spectrum and the quasiparticle energies obtained by many-body perturbation theory calculations [36]. Furthermore, ASIC appears to be a very suitable method to study molecules on surfaces: while it corrects the (occupied) molecular orbitals for the self-interaction error thus improving the overall description of the (occupied) molecular spectrum, it has only a minor effect on the delocalized metal states of the surface, which are usually well described by local and semilocal functionals. 
For the free molecules, cubic supercells with lateral dimension equal to $30 \AA$ were considered. For the fcc $(001)$ Co surface, we built a four-layer slab with a $(4 \times 4)$ square unit cell and each slab was separated by $60 \AA$ of vacuum from its periodic image. As fcc Co is grown epitaxially on a $\mathrm{Cu}$ substrate, the Co lattice constant was fixed to $3.61 \AA$, the experimental lattice constant of $\mathrm{Cu}$. Relaxations were performed with standard conjugate gradients until the forces were smaller than $0.04 \mathrm{eV} / \AA$. The two bottom layers of the Co slab were constrained and only the two top layers were allowed to relax. All SIESTA calculations were performed at the $\gamma$ point only.

Further calculations were also performed by using the FHI-AIMS all-electron code $[37,38]$ in order to achieve a larger accuracy, in particular concerning total energy calculations (all energy values presented in this paper were computed with FHI-AIMS according to the computational details provided here). We considered the standard numerical atom-centered orbitals basis set "tier2" for $\mathrm{H}, \mathrm{C}, \mathrm{N}, \mathrm{O}, \mathrm{Al}, \mathrm{Ga}$, and In and "tier1" for Co. The atomic zeroth-order regular approximation (ZORA) [37] was applied to treat relativistic effects (for all the molecules the energy difference between the results of "single-point" DFT calculations obtained with the atomic ZORA and the scaled ZORA [39] were found to be negligible). Like in the case of the SIESTA calculations, geometric relaxations were performed by using the PBE-GGA functional. Furthermore we also used the PBE $+\mathrm{vdW}^{\text {surf }}$ method [40] in order to investigate the effects of the van der Waals interactions on the absorption geometry. The screened $C_{6, L Z K}$ coefficient, the polarizability $\alpha_{L Z K}$, and the vdW radius $R_{L Z K}$ of Co were previously calculated [41] according to the scheme of Ruiz et al. [40] and they are equal to $C_{6, L Z K}=52.7$ hartree bohr ${ }^{3}$, $\alpha_{L Z K}=8.6$ bohr $^{3}$, and $R_{L Z K}=2.1 \mathrm{bohr}$ (the experimental dielectric constant for the hcp phase of Co [42] was used as this is expected not to differ substantially from that of the fcc phase). Although the PBE $+\mathrm{vdW}^{\text {surf }}$ method has been extensively tested for molecules both physisorbed and chemisorbed on metals $[40,43,44]$, the performances for ferromagnetic surfaces have never been assessed in detail. In fact no clear notion on how the van der Waals interactions are affected by the spin polarization has been established yet. Nevertheless we expect that the $\mathrm{PBE}+\mathrm{vdW}^{\text {surf }}$ may still represent an improvement over standard PBE, when the concerned effect of the van der Waals force cannot be neglected.

For the slab calculations, we tested various $k$-point meshes with the largest being $8 \times 8 \times 1$, but the results were well converged already for the $4 \times 4 \times 1$ mesh. The same supercell employed in the calculations performed with SIESTA was considered and atomic optimizations were carried out until forces were smaller than $0.01 \mathrm{eV} / \AA$. In order to compensate for the potential dipole field of the slab containing the molecules, we added an electrostatic potential step in the vacuum region.

Finally we remark that $\mathrm{Alq}_{3}$ has two isomers, commonly called facial and meridional (respectively with $C_{3}$ and $C_{1}$ symmetry). In agreement with other studies [45], we found the meridional isomer of $\mathrm{Alq}_{3}$ to be $0.175 \mathrm{eV}$ more stable than the facial one. This is also consistent with the fact that the facial isomer has only been detected in the high-temperature phase [46] and not in thin films [47]. Similarly, the meridional isomers of $\mathrm{Gaq}_{3}$ and $\mathrm{Inq}_{3}$ are calculated, respectively, as 0.192
TABLE I. Metal-O $\left(d_{\mathrm{M}-\mathrm{O}}\right)$ and Metal-N $\left(d_{\mathrm{M}-\mathrm{N}}\right)$ bond lengths for the meridional isomer of $\mathrm{Alq}_{3}, \mathrm{Gaq}_{3}$, and $\mathrm{Inq}_{3}$, as calculated from GGA-PBE.

\begin{tabular}{lcc}
\hline \hline Molecule & $d_{\text {M-O }}(\AA)$ & $d_{\text {M-N }}(\AA)$ \\
\hline $\mathrm{Alq}_{3}$ & $1.89,1.85,1.89$ & $2.09,2.05,2.08$ \\
$\mathrm{Gaq}_{3}$ & $2.01,1.97,2.0$ & $2.15,2.12,2.16$ \\
$\mathrm{Inq}_{3}$ & $2.18,2.15,2.18$ & $2.32,2.29,2.32$ \\
\hline \hline
\end{tabular}

and $0.165 \mathrm{eV}$ more stable than the facial ones. We have then decided to present the calculations only for this isomer. The metal-N and metal-O bond lengths are listed in Table I and, in the case of $\mathrm{Alq}_{3}$, they are within $1 \%$ of the results obtained in previous studies [45] (the tiny differences can also be attributed to the use of a different GGA functional as well as to a different numerical implementation of DFT).

\section{ELECTRONIC STRUCTURE OF THE Mq $\mathrm{M}_{3}$ MOLECULES}

We first discuss the electronic properties of the free $\mathrm{Mq}_{3}$ molecules. Figure 2 shows the UPS spectra of the $\mathrm{Co} / \mathrm{Mq}_{3}$ system, recorded for a molecular coverage of four monolayers $(\mathrm{ML} ; 1 \mathrm{ML}=1.3 \mathrm{~nm})$. The spectra in the right panel are plotted after subtraction of the secondary electron background using the procedure described in Ref. [48], while the left panel shows the low-energy cutoff region. For coverages above $4 \mathrm{ML}$, electrons originating from the cobalt and from the $\mathrm{Co} / \mathrm{Mq}_{3}$ interface cannot be detected anymore, since in UPS experiments the electron mean free path in $\mathrm{Mq}_{3}$ is considerably smaller than $5 \mathrm{~nm}\left(0.6 \mathrm{~nm}\right.$ for $\mathrm{Alq}_{3}$ [14]). This means that the molecular orbitals determined by UPS on this system can be compared to the occupied manifold of the free $\mathrm{Mq}_{3}$ molecules. Inspection of Fig. 2 reveals that the UPS spectra are almost

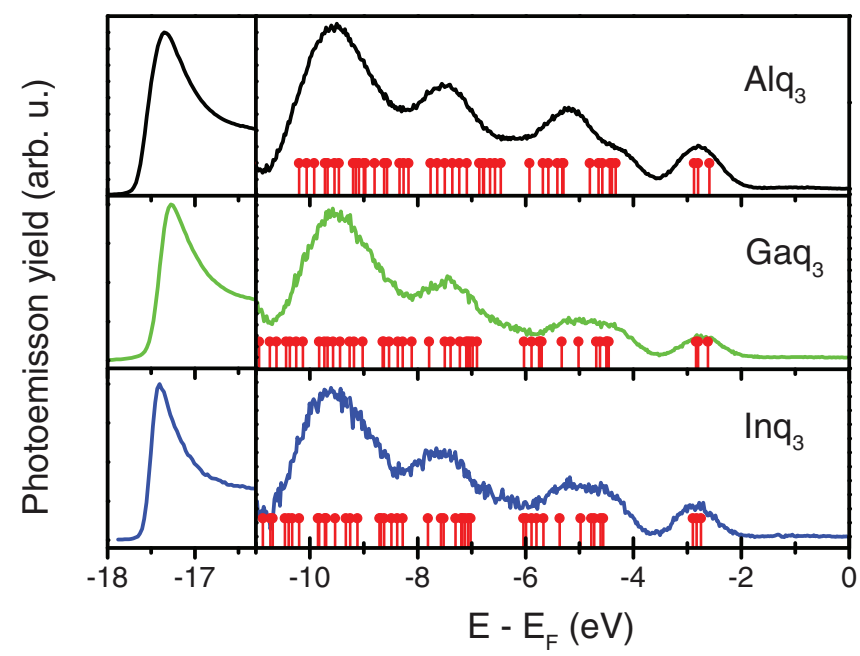

FIG. 2. (Color online) UPS spectrum for $\mathrm{Alq}_{3}$ (top panel), $\mathrm{Gaq}_{3}$ (middle), and $\mathrm{Inq}_{3}$ (bottom). Left panel: secondary electron cutoff; right panel: UPS spectrum after subtraction of the secondary electron background. The red ticks mark the position of the KS eigenvalues calculated with the ASIC scheme (the HOMO was aligned with experimental HOMO). 
identical for all three molecules. Moreover the spectrum of $\mathrm{Alq}_{3}$ matches very well the results of previous studies [49]. Regarding the highest occupied molecular orbitals (HOMOs) of the three molecules, we extract the following binding energies: $\mathrm{HOMO}\left(\mathrm{Alq}_{3}\right)=2.8 \mathrm{eV}, \mathrm{HOMO}\left(\mathrm{Gaq}_{3}\right)=2.7 \mathrm{eV}$, and $\mathrm{HOMO}\left(\mathrm{Inq}_{3}\right)=2.8 \mathrm{eV}$ (note that the energies are determined from the center of the HOMO peak and that the zero energy value is set at the Fermi level of the Co substrate). Figure 2 also displays the Kohn-Sham (KS) eigenvalues calculated with the ASIC functional (red ticks). Their position is in good agreement with the main peaks in the UPS spectra thus providing a further confirmation of the good performances of ASIC. Moreover, Fig. 2 also shows that, for all three compounds, the HOMO peaks are composed of three nearly degenerate molecular orbitals (note that these orbitals are not exactly degenerate because the molecule has no rotational symmetry). Therefore the positions of the UPS HOMO peak correspond to the center-of-mass position of these levels.

From the position of the low-energy cutoff in the UPS spectra (left panel of Fig. 2) we extract the work function $\Phi$ : $\Phi\left(\mathrm{Alq}_{3}\right)=3.6 \mathrm{eV}, \Phi\left(\mathrm{Gaq}_{3}\right)=3.7 \mathrm{eV}$, and $\Phi\left(\mathrm{Inq}_{3}\right)=3.6 \mathrm{eV}$ (see also Fig. 6). By adding the work function to the binding energy of the HOMO we obtain the experimental ionization potentials $\left(\mathrm{IP}_{\text {expt}}\right)$ of the three $\mathrm{Mq}_{3}$ molecules. These are reported in Table II and, in the case of $\mathrm{Alq}_{3}$, IP expt compares well with other estimates which give values between 6.3 and $6.5 \mathrm{eV}$ (see Ref. [50], and references therein).

From the theoretical side, quite accurate estimates for the IPs can usually be obtained by using the $\triangle \mathrm{SCF}$ method [51]. The results are reported in Table II, together with the computed electron affinity (EA), the absolute value of the total dipole moment $p$, and the value of the transport (or quasiparticle) gap $\Delta$, defined as the difference between the IP and the EA. Interestingly, IP, EA, and thus $\Delta$, are very similar for all three molecules, while the dipole moment changes along the series, with the largest belonging to $\mathrm{Alq}_{3}$. This seems to correlate to the increased metal-O $(\mathrm{N})$ bond length as seen in Table I. Furthermore, as a side comment, we note that the theoretically determined IP is within $2 \%$ of the experimental values. However, this observation must be taken with some caveats. In fact, first, the experimental HOMO values represent the center-of-mass position of the three almost degenerate occupied states, and it is thus not exactly the energy position of the real HOMO, which cannot be resolved because of the too small separation from the other states. Secondly, the computed values for IP refer to the molecule in the gas phase, while $\mathrm{IP}_{\text {expt }}$ is for molecules on a molecular thin-film surface. The gas-phase and the condensed-phase ionization potential

TABLE II. Experimental ( IP $_{\text {expt }}$ ) and computed ionization potential (IP), electron affinity (EA), transport gap $(\Delta)$, and absolute value of the dipole moment $(p)$ of the three molecules investigated. The computed ionization potential (electron affinity) is obtained by using the relaxed geometry for the positive (negative) ion.

\begin{tabular}{lccccc}
\hline \hline Molecule & $\mathrm{IP}_{\text {expt }}(\mathrm{eV})$ & $\mathrm{IP}(\mathrm{eV})$ & $\mathrm{EA}(\mathrm{eV})$ & $\Delta(\mathrm{eV})$ & $p(\mathrm{D})$ \\
\hline $\mathrm{Alq}_{3}$ & 6.4 & 6.29 & 1.23 & 5.06 & 4.04 \\
$\mathrm{Gaq}_{3}$ & 6.4 & 6.29 & 1.22 & 5.05 & 3.89 \\
$\mathrm{Inq}_{3}$ & 6.4 & 6.26 & 1.23 & 5.03 & 3.56 \\
\hline \hline
\end{tabular}

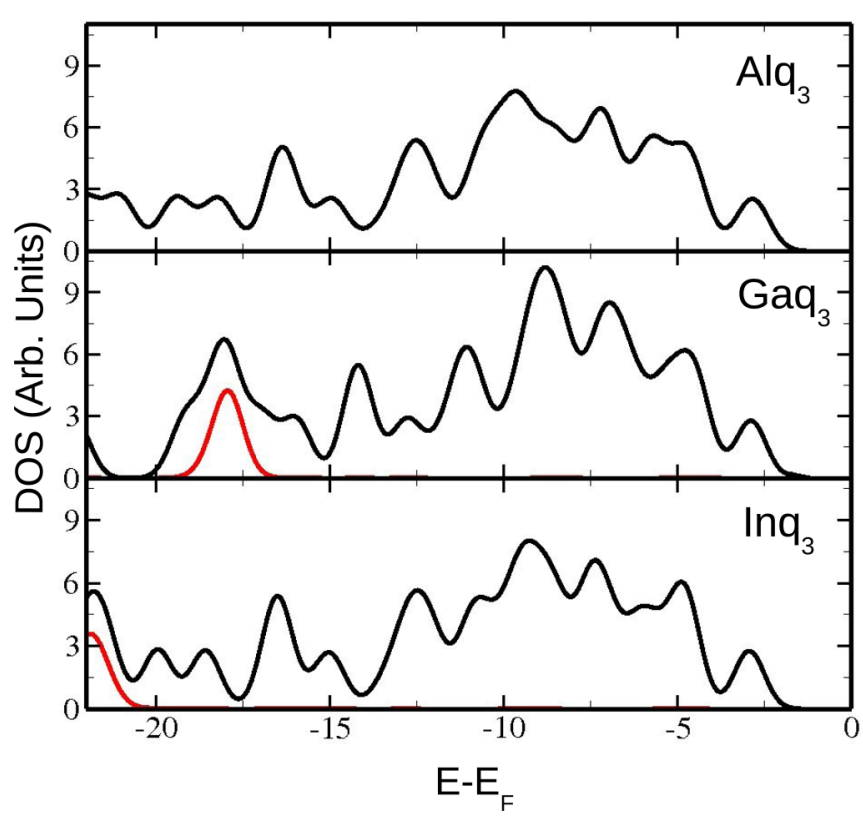

FIG. 3. (Color online) Calculated DOS (black line) for Alq ${ }_{3}$ (top panel), $\mathrm{Gaq}_{3}$ (middle), and $\mathrm{Inq}_{3}$ (bottom). The red lines represent the $3 d$-Ga and the $4 d$-In states. The spectrum was rigidly displaced in order to align the HOMO at the energy corresponding to $\Phi-$ IP (where IP is the computed ionization potential and $\Phi$ is the experimental work function determined from the low-energy cutoff). A Gaussian broadening was added to the computed DOS in order to better compare the theoretical and the experimental spectra in Fig. 2.

generally differ for the polarization energy, which has been estimated to be between 0.85 and $1 \mathrm{eV}$ for an $\mathrm{Alq}_{3}$ molecule on a crystal surface layer [52,53]. Therefore, it is fair to argue that the agreement between IP and IP expt in Table II comes from a cancellation of errors and IP is likely to be underestimated by about $0.9 \mathrm{eV}$ in our calculations.

Finally we investigate why the UPS spectra of the three molecules show very little differences. This can be better understood by looking at the DOS projected over the $3 d$-Ga orbitals of $\mathrm{Gaq}_{3}$ and the $4 d$-In of $\mathrm{Inq}_{3}$ (Fig. 3). In fact, we note that the orbitals of the metal centers contribute to the total DOS only for large binding energies (about $16 \mathrm{eV}$ for $\mathrm{Gaq}_{3}$ and $20 \mathrm{eV}$ for $\mathrm{Inq}_{3}$ ), while the states around the Fermi level are localized on the quinoline ligands. This is more clearly illustrated by Fig. 4, which shows the sum of the charge density associated with the three HOMOs and LUMOs. For all three molecules, the HOMOs (left panels of Fig. 4) are localized on the phenoxide moiety of the ligands. The three LUMOs (right panels of Fig. 4), in contrast, are localized on the pyridyl moiety.

\section{ELECTRONIC STRUCTURE OF THE INTERFACES}

We now move to investigate the electronic structure of the $\mathrm{Mq}_{3} / \mathrm{Co}$ interfaces by NT-PS and 2PPE. In order to obtain spectroscopic information about the interface, we have chosen a molecular coverage of $1 \mathrm{ML}$. At this coverage, the $\mathrm{Co} / \mathrm{Mq}_{3}$ interface is completely formed, while the $\mathrm{Mq}_{3}$ film is still thin enough for the interface features to be detected. Moreover, NT-PS has a deeper probing depth than UPS and is thus more suitable for detecting the interfacial electronic structure [54]. 
(a)

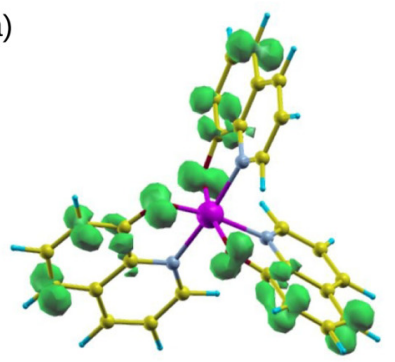

(b)

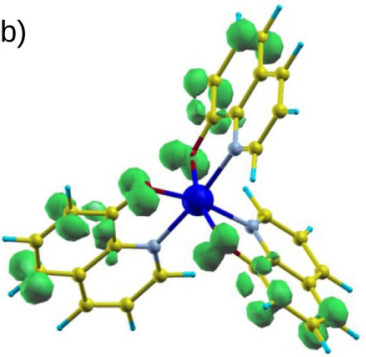

(c)

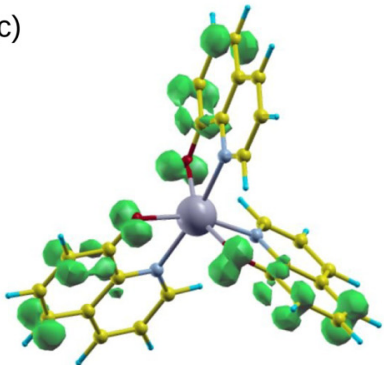

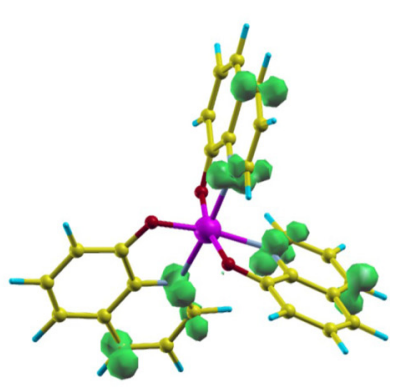
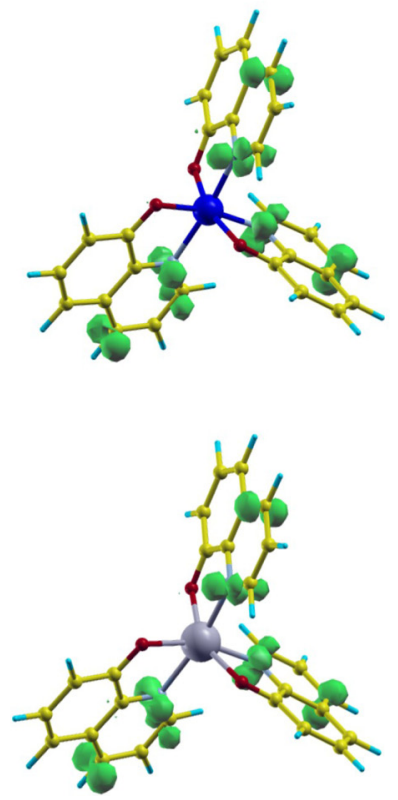

FIG. 4. (Color online) Isosurfaces for the sum of the total charge densities of the quasidegenerate HOMO, HOMO-1, and HOMO-2 (left panels) and of the LUMO, LUMO+1, and LUMO+2 levels (right panels) of $\mathrm{Alq}_{3}(\mathrm{a}), \mathrm{Gaq}_{3}$ (b), and $\mathrm{Inq}_{3}$ (c). Calculations are reported for the ASIC functional.

Figure 5(b) shows the NT-PS spectra of the three $\mathrm{Mq}_{3} / \mathrm{Co}$ interfaces. Note that in NT-PS experiments, we detect the occupied electronic states down to approximately $2.5 \mathrm{eV}$ below

the Fermi energy $E_{\mathrm{F}}$. Therefore only the onset of the HOMO is visible in the spectra at the low-energy cutoff. From the energetic position of the low-energy cutoff we also extract the value of the shift of the vacuum level due to the interface dipole. This is approximately $1.5 \mathrm{eV}$ for all three molecules and, in the case of $\mathrm{Alq}_{3}$, this is consistent with the value from a previous report [9]. For completeness in Fig. 5(a) we also show the UPS spectra in the HOMO region, recorded for the same system ( $1 \mathrm{ML} \mathrm{Mq}_{3}$ on $\mathrm{Co}$ ). The binding energy of the HOMO at the three $\mathrm{Co} / \mathrm{Mq}_{3}$ interfaces is $\mathrm{HOMO}\left(\mathrm{Co} / \mathrm{Alq}_{3}\right)=2.4 \mathrm{eV}$, $\mathrm{HOMO}\left(\mathrm{Co} / \mathrm{Gaq}_{3}\right)=2.4 \mathrm{eV}$, and $\mathrm{HOMO}\left(\mathrm{Co} / \mathrm{Inq}_{3}\right)=2.5 \mathrm{eV}$. Going back to the NT-PS spectra of Fig. 5(b), a second spectral feature is present in the spectra for all three molecules at a binding energy of $0.8 \mathrm{eV}$ for $\mathrm{Alq}_{3}, 0.9 \mathrm{eV}$ for $\mathrm{Gaq}_{3}$, and $1.1 \mathrm{eV}$ for $\mathrm{Inq}_{3}$. As such spectral features disappear from the NT-PS spectra for increasing $\mathrm{Mq}_{3}$ coverage, they are attributed to occupied hybrid interface states (oHISs), in agreement with our previous findings reported for $\mathrm{Alq}_{3}$ in Ref. [14]. In addition, we note that these hybrid interface states disappear after oxidation of the Co surface [55], thus demonstrating that they are related to the coupling between the surface and the molecules.

The 2PPE spectra after secondary electron background subtraction [14] are shown in Fig. 5(c). They are dominated by a very pronounced spectral feature, which can be ascribed to an unoccupied HIS (uHIS). The energetic position of the uHIS for the three molecules is, respectively, $\mathrm{uHIS}\left(\mathrm{Alq}_{3}\right)=$ $1.5 \mathrm{eV}, \mathrm{uHIS}\left(\mathrm{Gaq}_{3}\right)=1.4 \mathrm{eV}$, and $\mathrm{uHIS}\left(\mathrm{Inq}_{3}\right)=1.3 \mathrm{eV}$. The main results of the NT-PS and 2PPS experiments are further summarized through the diagrams of Fig. 6, which illustrate the energy level alignment at the $\mathrm{Mq}_{3} / \mathrm{Co}$ interfaces.

The origin of the observed hybrid interface states can be better understood by performing DFT calculations. The GGA lowest energy geometry for $\mathrm{Alq}_{3}$ on $\mathrm{Co}$ is displayed in Fig. 7 (the results for $\mathrm{Gaq}_{3}$ and $\mathrm{Inq}_{3}$ are very similar and, therefore, are not shown here). The molecule is adsorbed with two $\mathrm{O}$ and one $\mathrm{N}$ atom pointing toward the Co surface (a configuration sometimes referred to as "up" [56]). Then, after the geometry optimization, the angle in between the two quinoline ligands closer to the surface opens until these ligands lay down on the surface, while the third ligand remains almost (a)

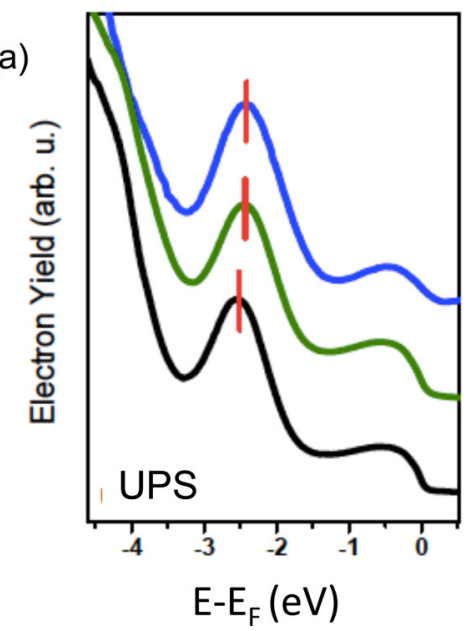

(b)

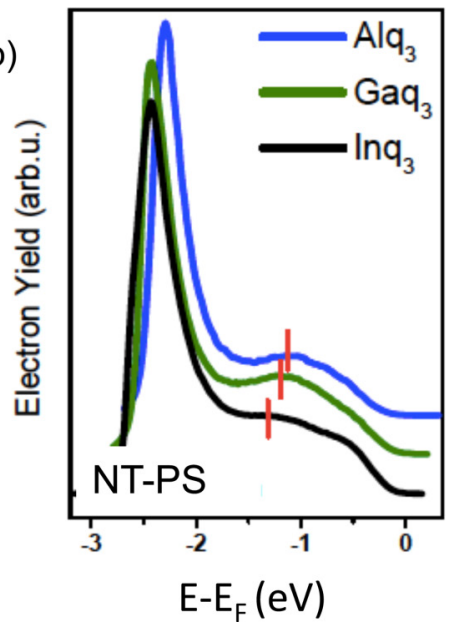

(c)

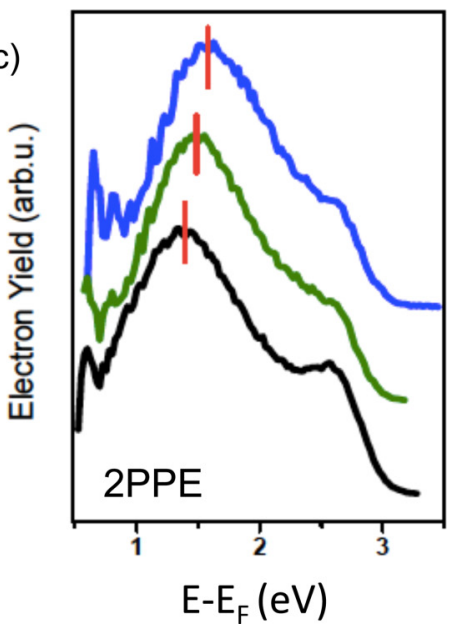

FIG. 5. (Color online) (a) UPS, (b) NT-PS, and (c) 2PPE spectra (after background subtraction) of one monolayer of Mq ${ }_{3}$ on Co, from which we extract the occupied and unoccupied electronic structure of the $\mathrm{Co} / \mathrm{Mq}_{3}$ interface. 


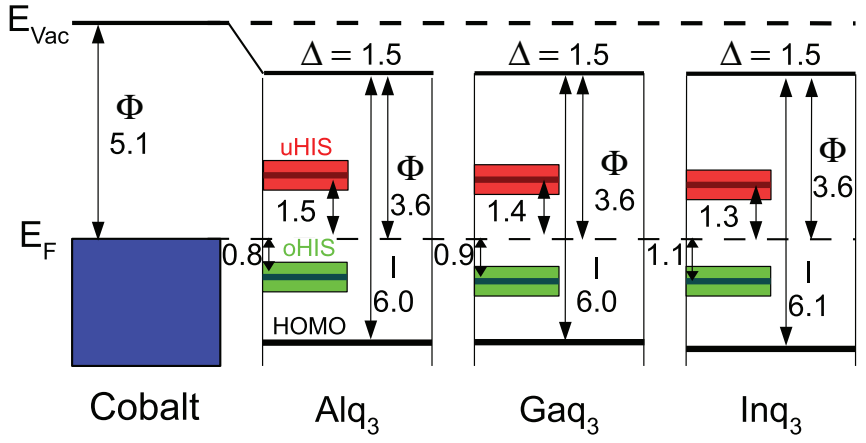

FIG. 6. (Color online) Diagram of the energy level alignment for the three $\mathrm{Mq}_{3} / \mathrm{Co}$ interfaces as extracted from the NT-PS, UPS, and $2 \mathrm{PPE}$ experiments for the $\mathrm{Co} / \mathrm{Mq}_{3}$ interface ( $1 \mathrm{ML} \mathrm{Mq}_{3}$ on $\mathrm{Co}$ ) in Fig. 5.

perpendicular to the interface. As such the molecule becomes strongly chemisorbed through the hybridization between the $d$-Co states and the $p_{z}$ orbitals of the ligands. As an additional test, we have also verified that the inclusion of the van der
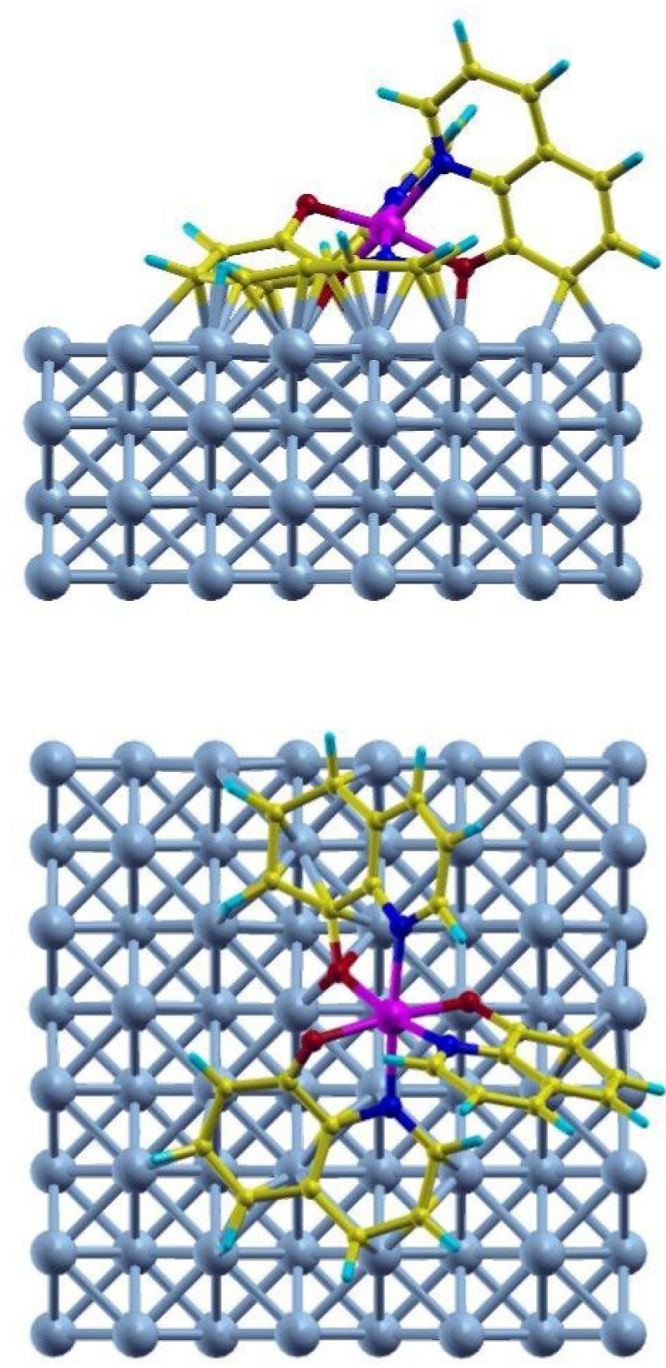

FIG. 7. (Color online) Top view and lateral view of the $\mathrm{Alq}_{3} / \mathrm{Co}$ interface after the DFT geometry optimization. Color code: C (yellow), $\mathrm{O}$ (red), N (gray), $\mathrm{H}$ (blue), and $\mathrm{Al}$ (purple).
Waals interactions through the $\mathrm{PBE}+\mathrm{vdW}$ surf functional does not affect the final geometry for such a strongly absorbed system (although the binding energy is expected to change significantly [43]).

In addition to the "up" geometry, we have also considered a second configuration (sometimes called "down" [56]) with two $\mathrm{N}$ atoms and one $\mathrm{O}$ atom pointing toward the Co surface. However, our structural optimization was unable to reach a stable energy minimum and, ultimately, the two quinoline ligands tended to be strongly bonded to the surface and to detach from the central metal ion, namely, the molecule gets fragmented.

Notably, the optimized geometry presented in Fig. 7 is very similar to the results of Zhan et al. [57] for $\mathrm{Alq}_{3}$ on $\mathrm{Fe}$. In that case the strong interaction between the molecule and the surface was further demonstrated by angle-resolved $\mathrm{N}(1 \mathrm{~s}) \mathrm{x}$-ray photoelectron spectroscopy. In fact, in that paper the presence of three peaks in the spectrum (two with lower binding energies than the third one) suggests that two ligands are located very close to the surface with the third being relatively far and only weakly coupled to the surface.

Having obtained the optimized geometry, we have computed the work function of the surface. This is evaluated as the difference between the Fermi energy and the reference Hartree potential in the vacuum region far away from the surface. We find a value of $5.2 \mathrm{eV}$ for $\mathrm{Co}$ and $3.7 \mathrm{eV}$ for all three $\mathrm{Co} / \mathrm{Mq}_{3}$ combined systems (note that at least ten layers of cobalt in the supercell and the inclusion of an electrostatic potential step in the vacuum region are required in order to obtain well converged results). Thus the computed shifts of the vacuum level due to the interface dipole $(\Delta=1.5 \mathrm{eV})$ are virtually identical to the experimental estimates and they have the same value for all three cases, although the three molecules were predicted to have slightly different dipole moments in the gas phase (see Table II). Note that, after absorption, the molecules are so strongly coupled to the surface, that they lose their gas-phase molecular features and, therefore, a prediction of the work-function shift based solely on the inspection of the gas-phase $\mathrm{Mq}_{3}$ dipole is not appropriate. Furthermore, even if it is not possible to disentangle the relative contribution of the various effects determining the Co work-function shift, we argue that the non-negligible molecule-Co charge transfer plays an important role. As this charge transfer is the same for all three molecules, it is reasonable to expect a similar change for the work function.

Although the geometry in Fig. 7 accounts for the strong chemisorption of $\mathrm{Alq}_{3}$ (as well as $\mathrm{Gaq}_{3}$ and $\mathrm{Inq}_{3}$ ) on $\mathrm{Co}$, it corresponds only to a partial surface coverage. Furthermore, $\mathrm{Mq}_{3}$ molecules do not self-assemble in an ordered way on Co so that a meaningful simulation of the UPS spectra can only be carried out by performing an average over several possible absorption configurations. In this respect and in order to describe one full ML, we have embedded a second molecule in the supercell (see Fig. 8). As this second molecule can be placed in many different ways, we have considered a limited representative ensemble of all possible two-molecule configurations and we have then performed a careful optimization by including the van der Waals interaction (according to what was discussed by Egger et al. [58]). Then, while the first quinoline is chemisorbed, the second is just physisorbed or very weakly chemisorbed as not enough "clean" Co remains 


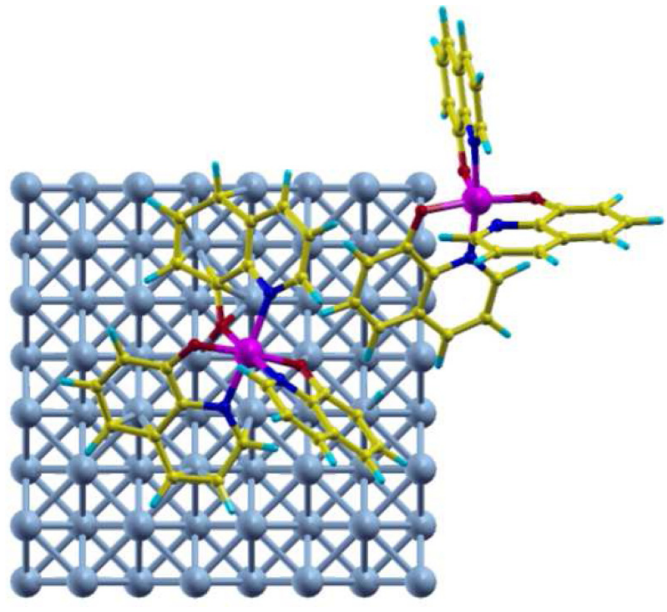

FIG. 8. (Color online) Top view of one of several supercells containing two $\mathrm{Alq}_{3}$ molecules that have been investigated in this work. The color code for the various atoms is the same as that of Fig. 7.

available on the surface for the second molecules to bind. Interestingly, we have verified that the inclusion of the second weakly coupled molecule does not affect the computed change in work function, in spite of the large dipole moment of the three compounds. This confirms that the electronic structure of the interface is entirely determined by the chemical bond of the first molecule.

The DOS for the $\mathrm{Co} / \mathrm{Alq}_{3}$ system computed with ASIC is displayed in Fig. 9, together with the UPS spectrum already presented in Fig. 5(a). The computed DOS has been obtained by averaging over several two-molecule supercells (after subtracting the contribution of all cobalt atoms) and was convoluted with a Fermi function in order to simulate the tailing off of the UPS spectrum at the Fermi level. We observe a remarkably good agreement between the theoretical calculations and the UPS spectrum (the results for $\mathrm{Gaq}_{3} / \mathrm{Co}$ and

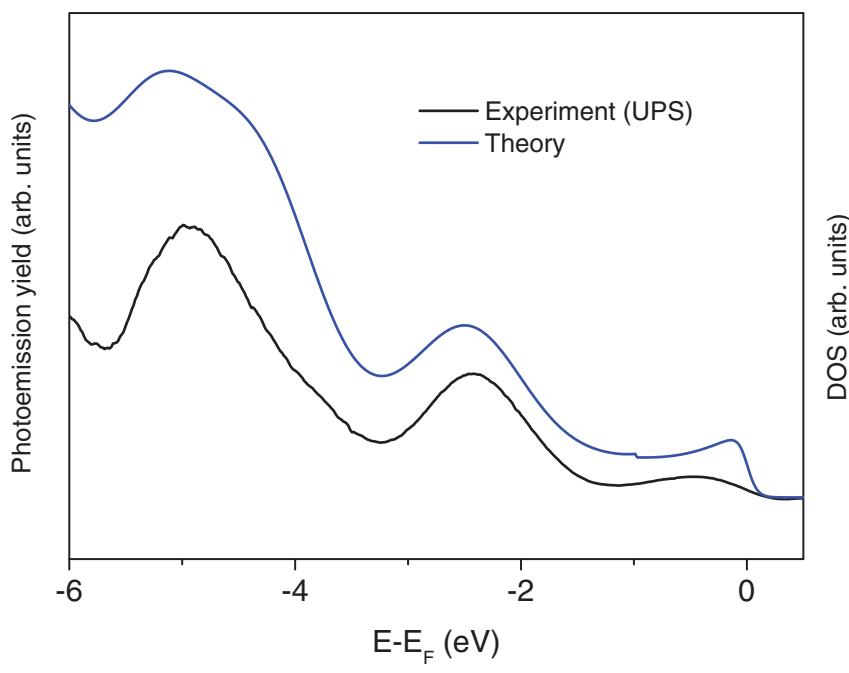

FIG. 9. (Color online) UPS spectrum and total DOS for one monolayer of $\mathrm{Alq}_{3}$. The DOS is the result of the average over several two-molecule configurations, such as that in Fig. 8, and with the contribution from the cobalt atoms subtracted.
$\mathrm{Inq}_{3} / \mathrm{Co}$ are identical and therefore they are not shown here). In particular, in the ASIC DOS, both the oHIS and the HOMO are recognizable (note, however, that no renormalization of the HOMO energy due to charge image effects [53,59-62] has been considered so that a careful analysis of the HOMO position shift is not possible). While the oHIS originates from the hybridization between the chemisorbed molecules and the surface, the HOMO of the weakly coupled molecules dominates the spectrum below $-2 \mathrm{eV}$. This explains why, except for the oHIS and the shift of the HOMO to lower energies, no marked differences between the UPS spectrum of $1 \mathrm{ML} \mathrm{Co} / \mathrm{Alq}_{3}$ and $4 \mathrm{ML} \mathrm{Co} / \mathrm{Alq}_{3}$ are observed.

Finally, although in the present work we limit ourselves to computing occupied states only (thus leaving the quite complex theoretical discussion of the unoccupied spectrum for future studies), we must add a remark concerning the small difference observed in the 2PPE spectrum of the three systems. Although $\mathrm{Inq}_{3}$ is predicted to relax similarly to $\mathrm{Alq}_{3}$ and $\mathrm{Gaq}_{3}$, the In ion ends up slightly closer to the surface than both the $\mathrm{Al}$ and $\mathrm{Ga}$ ions. This leads the $\mathrm{N}$ atom, which points toward the surface, to form a bond stronger for $\mathrm{Inq}_{3}$ than for $\mathrm{Alq}_{3}$ and $\mathrm{Ga}_{3}$. As the unoccupied hybrid states are likely to originate from the $\mathrm{Mq}_{3}$ LUMOs, which are localized on the pyridyl side of the quinoline group, this stronger bond may be reflected in the lower energy of the $\mathrm{Inq}_{3} / \mathrm{Co}$ uHIS than that of the $\mathrm{Alq}_{3} / \mathrm{Co}$ and $\mathrm{Gaq}_{3} /$ Co hybrid states.

\section{SPIN POLARIZATION AT THE INTERFACES}

As the HISs originate from the coupling between the molecules and the ferromagnetic Co surface, they present a non-negligible spin polarization, which we detected by spin-resolved (SR) NT-PS and SR-2PPE, for occupied and unoccupied, respectively. In order to quantify the spin properties of interface states, we define the relative spin polarization as the ratio between the spin polarization of the cobalt and the spin polarization of the $\mathrm{Co} / \mathrm{Mq}_{3}$ interface. The spin polarization is defined as

$$
P=\frac{N^{\uparrow}-N^{\downarrow}}{N^{\uparrow}+N^{\downarrow}},
$$

where $N^{\uparrow}$ and $N^{\downarrow}$ are, respectively, the spin-resolved majority and minority electron spectra as detected by spin-resolved photoemission (note that we measure the spin component along the direction of the cobalt easy axis and that the definition of majority and minority electrons is the same for the cobalt substrate and the $\mathrm{Co} / \mathrm{Mq}_{3}$ interfaces). The shape and absolute value of the relative spin polarization gives thus direct information about the changes in the spin polarization of the cobalt film arising from the formation of hybrid interface states.

The relative spin polarization measured at the $\mathrm{Co} / \mathrm{Mq}_{3}$ interface by SR-NT-PS and SR-2PPE is shown in Figs. 10(a) and 10(b), respectively. Also here, we observe a virtually identical behavior for the three molecules. In particular, the relative spin polarization measured by NT-PS is enhanced to 1.1 at the energetic position of the oHIS (around a binding energy of $0.8 \mathrm{eV}$ ), which means that the oHIS has a spin polarization larger than the underlying cobalt substrate. This can be better understood by looking at the computed magnetization density, i.e., the difference between the spin 

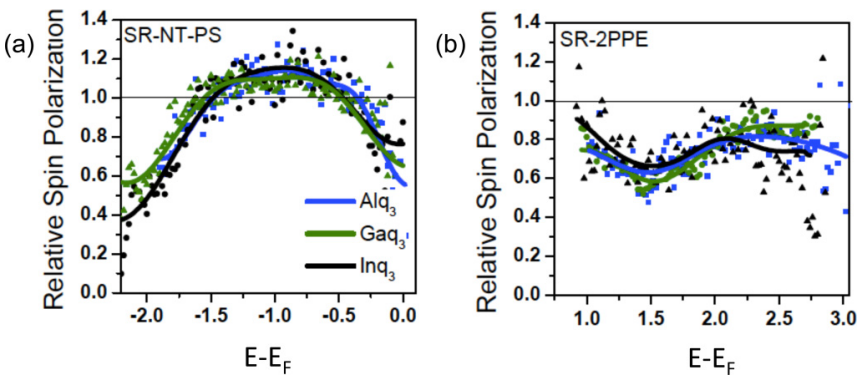

FIG. 10. (Color online) Relative spin polarization of the Co/ $\mathrm{Mq}_{3}$ interface as measured by (a) spin-resolved NT-PS and (b) spinresolved 2PPE (b). Solid lines are a guide to the eyes obtained by smoothing the experimental data (symbols).

up and spin down electron density (Fig. 11). In fact, the hybridization induces a large magnetization on the molecule. Specifically, after the absorption, the oxygen atoms acquire a magnetic moment parallel to that of Co surface, while the $\pi$-cojugated carbon atoms have an opposite (and smaller) spin polarization, similar to that imaged by means of spin-polarized scanning tunneling microscopy in the case of small aromatic molecules [18]. Thus, since the oHIS is derived mainly from the molecules' HOMOs, which receive a large contribution from the $p-\mathrm{O}$ states (see Sec. IV and Fig. 4), the magnetic moment induced on the oxygen atoms accounts for the observed enhanced relative spin- polarization at a binding energy of about $0.8 \mathrm{eV}$.

In contrast, the relative spin polarization measured by 2PPE drops to a value of 0.6 at the energetic position of the uHIS (around $1.5 \mathrm{eV}$ intermediate state energy). This, once again, can be partially understood by looking at the relative magnetization reported in Fig. 11. In fact, the uHIS is expected to be derived from the molecules' LUMOs, which are localized

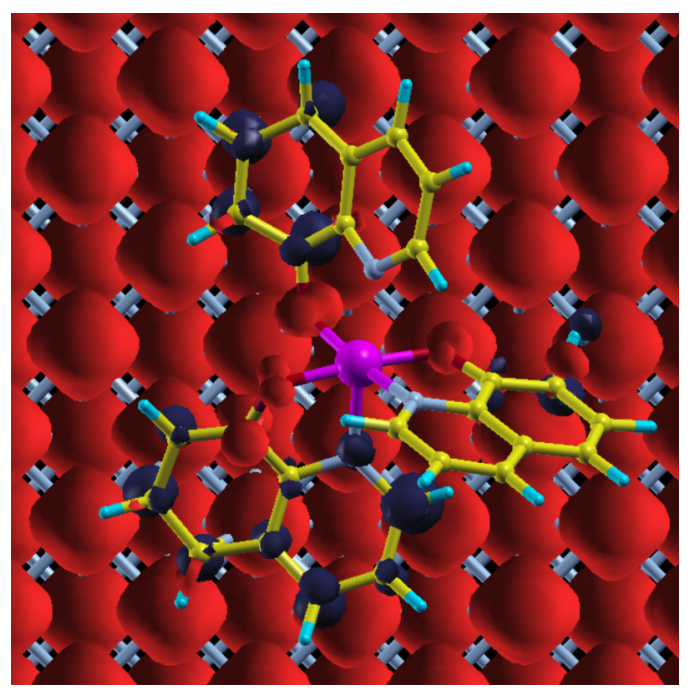

FIG. 11. (Color online) Computed magnetization density for $\mathrm{Co} / \mathrm{Alq}_{3}$. Red (purple) isosurfaces represent positive (negative) values of the magnetization. The results for $\mathrm{Co} / \mathrm{Gaq}_{3}$ and $\mathrm{Co} / \mathrm{Inq}_{3}$ are identical and not shown. The color code for the various atoms is the same as that of Fig. 7. on the pyridyl side of the ligands (see Fig. 4 and also Ref. [63]). Then after absorption, the net spin polarization of the nitrogen atoms, which are opposite to that of the cobalt surface, reduces the relative spin polarization of the interface.

Although based on this argument we can provide a qualitative explanation of the experimental results, we must remark that, differently from the case of the oHIS, the interpretation of the relative spin polarization of an unoccupied state is not at all trivial. In Ref. [14] some of us have shown that the uHIS of the $\mathrm{Co} / \mathrm{Alq}_{3}$ interface has a spin-dependent lifetime. More specifically, it was found that the lifetime of the minority electrons $(\approx 800 \mathrm{fs})$ is roughly twice the lifetime of the majority electrons. This means that during the 2PPE process (the pulse time length is $63 \mathrm{fs}$ ) majority electrons will depopulate the uHIS faster than the minority electrons, thus contributing to the measured effective reduction of the SP. Furthermore, we may need to consider the disorder in the single monolayer, which results in different absorption geometries for the molecules (see Fig. 8 and related discussion about the full coverage). Finally, there may be states originating from molecules which are more strongly bounded to the cobalt substrate than the uHIS: Such states would possess much shorter lifetimes than those detected in Ref. [14] and accordingly a much broader homogeneous linewidth. Such states have not been addressed in Ref. [14] (as extracting their lifetime is not trivial), but could possibly affect the relative spin polarization reported in Fig. 10(b).

These considerations highlight how complex the task of addressing the spin properties of unoccupied hybrid states is, thus suggesting that spin-, time-, and lateral-resolved spectroscopy experiments, as well as theoretical methods that allow for a reliable quantitative description of unoccupied states, are required in future studies in order to get a deeper insight into the problem.

\section{CONCLUSION}

In summary, the electronic properties of the interface between cobalt and the three molecules $\mathrm{Alq}_{3}, \mathrm{Gaq}_{3}$, and $\mathrm{Inq}_{3}$, have been investigated both experimentally and theoretically. The strong hybridization between the molecules and the metal surface induces occupied and unoccupied hybrid states, which present a net spin polarization and thus spinterface properties.

A thorough analysis demonstrates that the three $\mathrm{Co} / \mathrm{Mq}_{3}$ interfaces have very similar spin-dependent electronic properties. The small changes between the different interfaces can be explained by the fact that the HOMOs and the LUMOs of all three molecules are mainly localized on the quinoline ligands. This establishes that, in contrast to the case of other metal-organic complexes, the electronic and magnetic properties of the interface between $\mathrm{Mq}_{3}$ molecules and cobalt cannot be modified by chemically substituting the metal ion.

\section{ACKNOWLEDGMENT}

The research leading to these results was financially supported by the EU project NMP3-SL-2011-263104 HINTS and by the SFB/TRR 88 3MET from the DFG. 
[1] V. Dediu, M. Murgia, F. C. Matacotta, C. Taliani, and S. Barbanera, Solid State Commun. 122, 181 (2002).

[2] V. Dediu, L. E. Hueso, I. Bergenti, and C. Taliani, Nat. Mater. 8, 707 (2009).

[3] S. Sanvito, Chem. Soc. Rev. 40, 3336 (2011).

[4] Z. H. Xiong, D. Wu, Z. Valy Vardeny, and J. Shi, Nature (London) 427, 821 (2004).

[5] H. Ishii, K. Sugiyama, E. Ito, and K. Seki, Adv. Mater. 11, 605 (1999).

[6] J. Hwang, A. Wan, and A. Kahn, Mater. Sci. Eng., R 64, 1 (2009).

[7] S. Braun, W. R. Salaneck, and M. Fahlman, Adv. Mater. 21, 1450 (2009).

[8] Y. Q. Zhan, I. Bergenti, L. E. Hueso, V. Dediu, M. P. de Jong, and Z. S. Li, Phys. Rev. B 76, 045406 (2007).

[9] Y. Q. Zhan, M. P. de Jong, F. H. Li, V. Dediu, M. Fahlman, and W. R. Salaneck, Phys. Rev. B 78, 045208 (2008).

[10] M. Cinchetti, K. Heimer, J.-P. Wüstenberg, O. Andreyev, M. Bauer, S. Lach, C. Ziegler, Y. Gao, and M. Aeschlimann, Nat. Mater. 8, 115 (2009).

[11] A. J. Drew, J. Hoppler, L. Schulz, F. L. Pratt, P. Desai, P. Shakya, T. Kreouzis, W. P. Gillin, A. Suter, N. A. Morley, V. K. Malik, A. Dubroka, K. W. Kim, H. Bouyanfif, F. Bourqui, C. Bernhard, R. Scheuermann, G. J. Nieuwenhuys, T. Prokscha, and E. Morenzoni, Nat. Mater. 8, 109 (2009).

[12] C. Barraud, P. Seneor, R. Mattana, S. Fusil, K. Bouzehouane, C. Deranlot, P. Graziosi, L. Hueso, I. Bergenti, V. Dediu, F. Petroff, and A. Fert, Nat. Phys. 6, 615 (2010).

[13] S. Sanvito, Nat. Phys. 6, 562 (2010).

[14] S. Steil, N. Großmann, M. Laux, A. Ruffing, D. Steil, M. Wiesenmayer, S. Mathias, O. L. A. Monti, M. Cinchetti, and M. Aeschlimann, Nat. Phys. 9, 242 (2013).

[15] S. Lach, A. Altenhof, K. Tarafder, F. Schmitt, Md. E. Ali, M. Vogel, J. Sauther, P. M. Oppeneer, and C. Ziegler, Adv. Funct. Mater. 22, 989 (2012).

[16] F. Djeghloul, F. Ibrahim, M. Cantoni, M. Bowen, L. Joly, S. Boukari, P. Ohresser, F. Bertran, P. Le Fèvre, P. Thakur, F. Scheurer, T. Miyamachi, R. Mattana, P. Seneor, A. Jaafar, C. Rinaldi, S. Javaid, J. Arabski, J.-P. Kappler, W. Wulfhekel, N. B. Brookes, R. Bertacco, A. Taleb-Ibrahimi, M. Alouani, E. Beaurepaire, and W. Weber, Sci. Rep. 3, 1272 (2013).

[17] J. Brede, N. Atodiresei, S. Kuck, P. Lazic, V. Caciuc, Y. Morikawa, G. Hoffmann, S. Blugel, and R. Wiesendanger, Phys. Rev. Lett. 105, 047204 (2010).

[18] N. Atodiresei, J. Brede, P. Lazic, V. Caciuc, G. Hoffmann, R. Wiesendanger, and S. Blugel, Phys. Rev. Lett. 105, 066601 (2010).

[19] T. Methfessel, S. Steil, N. Baadji, N. Großmann, K. Koffler, S. Sanvito, M. Aeschlimann, M. Cinchetti, and H. J. Elmers, Phys. Rev. B 84, 224403 (2011).

[20] S. Müller, S. Steil, A. Droghetti, N. Großmann, S. Sanvito, V. Meded, A. Magri, B. Schäfer, O. Fuhr, M. Ruben, M. Cinchetti, and M. Aeschlimann, New J. Phys. 15, 113054 (2013).

[21] L. Schulz, L. Nuccio, M. Willis, P. Desai, P. Shakya, T. Kreouzis, V. K. Malik, C. Bernhard, F. L. Pratt, N. A. Morley, A. Suter, G. J. Nieuwenhuys, T. Prokscha, E. Morenzoni, W. P. Gillin, and A. J. Drew, Nat. Mater. 10, 39 (2011).
[22] M. Cinchetti, S. Neuschwander, A. Fischer, A. Ruffing, S. Mathias, J.-P. Wüstenberg, and M. Aeschlimann, Phys. Rev. Lett. 104, 217602 (2010).

[23] L. Nuccio, M. Willis, L. Schulz, S. Fratini, F. Messina, M. D’Amico, F. L. Pratt, J. S. Lord, I. McKenzie, M. Loth, B. Purushothaman, J. Anthony, M. Heeney, R. M. Wilson, I. Hernandez, M. Cannas, K. Sedlak, T. Kreouzis, W. P. Gillin, C. Bernhard, and A. J. Drew, Phys. Rev. Lett. 110, 216602 (2013).

[24] G. Gahungu and J. Zhang, J. Phys. Chem. B 109, 17762 (2005).

[25] P. Shakya, P. Desai, M. Somerton, G. Gannaway, T. Kreouzis, and W. P. Gillin, J. Appl. Phys. 103, 103715 (2008).

[26] P. E. Burrows, L. S. Sapochak, D. M. McCarty, S. R. Forrest, and M. E. Thompson, Appl. Phys. Lett. 64, 2718 (1994).

[27] O. Andreyev, Y. Koroteev, M. Sánchez Albaneda, M. Cinchetti, G. Bihlmayer, E. V. Chulkov, J. Lange, F. Steeb, M. Bauer, P. M. Echenique, S. Blügel, and M. Aeschlimann, Phys. Rev. B 74, 195416 (2006).

[28] H. Schmidbaur, J. Lettenbauer, D. L. Wilkinson, G. Muller, and O. Kumberger, Naturforsch. 46, 901 (1991).

[29] J. N. Petronio and W. E. Ohnesorge, Anal. Chem. 39, 460 (1967).

[30] M. Cinchetti, J.-P. Wüstenberg, M. Sánchez-Albaneda, F. Steeb, A. Conca, M. Jourdan, and M. Aeschlimann, J. Phys. D: Appl. Phys. 40, 1544 (2007).

[31] J. M. Soler, E. Artacho, J. D. Gale, A. García, J. Junquera, P. Ordejón, and D. Sánchez-Portal, J. Phys.: Condens. Matter 14, 2745 (2002).

[32] J. P. Perdew, K. Burke, and M. Ernzerhof, Phys. Rev. Lett. 77, 3865 (1996).

[33] J. P. Perdew, K. Burke, and M. Ernzerhof, Phys. Rev. Lett. 78, 1396 (1997).

[34] C. D. Pemmaraju, T. Archer, D. Sanchez-Portal, and S. Sanvito, Phys. Rev. B 75, 045101 (2007).

[35] A. Filippetti, C. D. Pemmaraju, S. Sanvito, P. Delugas, D. Puggioni, and V. Fiorentini, Phys. Rev. B 84, 195127 (2011).

[36] A. Droghetti and S. Sanvito (unpublished).

[37] V. Blum, R. Gehrke, F. Hanke, P. Havu, V. Havu, X. Ren, K. Reuter, and M. Scheffler, Comput. Phys. Commun. 180, 2175 (2009).

[38] V. Havu, V. Blum, P. Havu, and M. Scheffler, J. Comput. Phys. 228, 8367 (2009).

[39] E. van Lenthe, E. J. Baerends, and J. G. Snijders, J. Chem. Phys. 101, 9783 (1994).

[40] V. G. Ruiz, W. Liu, E. Zojer, M. Scheffler, and A. Tkatchenko, Phys. Rev. Lett. 108, 146103 (2012).

[41] A. Droghetti and S. Sanvito (unpublished).

[42] W. Werner, K. Glantschnig, and C. Ambrosch-Draxl, J. Phys. Chem. Ref. Data 38, 1013 (2009).

[43] W. Liu, J. Carrasco, B. Santra, A. Michaelides, M. Scheffler, and A. Tkatchenko, Phys. Rev. B 86, 245405 (2012).

[44] W. Liu, A. Savara, X. Ren, W. Ludwig, K.-H. Dostert, S. Schauermann, A. Tkatchenko, H.-J. Freund, and M. Scheffler, J. Phys. Chem. Lett. 3, 582 (2012).

[45] A. Curioni, M. Boero, and W. Andreoni, Chem. Phys. Lett. 294, 263 (1998)

[46] Z. Jian, Y. Luo, J. Chung, S. Tang, M. Kuo, J. Shen, K. Chiu, C. Yang, W. Chou, C. Dai, and J. Yeh, J. Appl. Phys. 101, 123708 (2007).

[47] R. J. Curry, W. P. Gillin, J. Clarkcon, and D. N. Batcherlder, J. Appl. Phys. 92, 1902 (2002). 
[48] V. E. Henrich. X. Li, and Z. Zhang, J. Electron. Spectrosc. Relat. Phenom. 63, 253 (1993).

[49] F. Bisti, A. Stroppa, M. Donarelli, S. Picozzi, and L. Ottaviano, Phys. Rev. B 84, 195112 (2011).

[50] I. G. Hill, A. Kahn, J. Cornil, D. A. dos Santos, and J. L. Bredas, Chem. Phys. Lett. 317, 444 (2000).

[51] R. O. Jones and O. Gunnarsson, Rev. Mod. Phys. 61, 689 (1989). We remind one that $I E=E(N-1)-E(N)$ and $E A=$ $E(N)-E(N+1)$, where $E(N), E(N-1)$, and $E(N+1)$ are the total energies of the neutral molecule and of the positively and negatively charged ions, respectively. In this work, these energies were computed with the PBE functional for their respective optimized geometries, i.e., $E A$ and $I E$ are for adiabatic transitions.

[52] I. G. Hill, A. Kahn, Z. G. Soos, and R. A. Pascal, Jr., Chem. Phys. Lett. 327, 181 (2000).

[53] I. G. Hill, A. J. Mäkinen, and Z. H. Kafafi, J. Appl. Phys. 88, 889 (2000).

[54] R. Fetzer, J.-P. Wüstenberg, T. Taira, T. Uemura, M. Yamamoto, M. Aeschlimann, and M. Cinchetti, Phys. Rev. B 87, 184418 (2013).
[55] N. Haag, S. Steil, N. Großmann, R. Fetzer, M. Cinchetti, and M. Aeschlimann, Appl. Phys. Lett. 103, 251603 (2013).

[56] S. Yanagisawa, K. Lee, and Y. Morikawa, J. Chem. Phys. 128, 244704 (2008).

[57] Y. Zhan, E. Holmström, R. Lizarraga, O. Eriksson, X. Liu, F. Li, E. Carlegrim, S. Stafstrom, and M. Fahlman, Adv. Mater. 22, 1626 (2010).

[58] D. A. Egger, V. G. Ruiz, W. A. Saidi, T. Bucko, A. Tkatchenko, and E. Zojer, J. Phys. Chem. C 117, 3055 (2013).

[59] E. V. Tsiper, W. Gao, Z. G. Soos, and A. Kahn, Chem. Phys. Lett. 360, 47 (2002).

[60] J. B. Neaton, M. S. Hybertsen, and S. G. Louie, Phys. Rev. Lett. 97, 216405 (2006).

[61] J. M. Garcia-Lastra, C. Rostgaard, A. Rubio, and K. S. Thygesen, Phys Rev. B 80, 245427 (2009).

[62] A. M. Souza, I. Rungger, C. D. Pemmaraju, U. Schwingenschloegl, and S. Sanvito, Phys. Rev. B 88, 165112 (2013).

[63] K. Tarafder, B. Sanyal, and P. M. Oppeneer, Phys. Rev. B 82, 060413(R) (2010). 